\title{
Creep crack growth assessment in terms of continuum damage mechanics
}

\author{
Valery Shlyannikov ${ }^{1, *}$ and Andrey Tumanov ${ }^{1}$ \\ ${ }^{1}$ Kazan Scientific Center, Russian Academy of Sciences, 420111 Kazan, Russia
}

\begin{abstract}
The stress, strain rate, and process zone with respect to the creep-crack growth in compacttension $\mathrm{C}(\mathrm{T})$ specimen is analyzed by employing damage-evolution equations. The damage model for the fracture of the process zone is represented using a stress based formulation. Both damage free and defective creeping solids have been studied. The variations in the creep stress/strain and crack-tip governing parameter in terms of the creep stress intensity factors with respect to time and the evolution of creep damage are analyzed using an FE model for $\mathrm{C}(\mathrm{T})$ specimen. The creep-fatigue crack growth rate tests were performed on special designed program test-cycle. The interpretation of the experimental creep crack growth rate data was given in terms of introduced creep stress intensity factors based on undamaged and damaged stress/strain fields.
\end{abstract}

\section{Introduction}

In the past, the continuum damage mechanics (CDM) was used as a complementary approach in hightemperature fracture mechanics to analyze the damage and fracture process at different scales with respect to the changing material structure from the initiation of micro-cracks to the final fracture resulting from the macro-cracks [1, 2]. The CDM covered several microscopic mechanisms in the area near the macroscopic creep crack including void nucleation and growth on the grain-boundary facets, cavities coalescing to form a grain-size micro-crack, coalescing of microcracks, which finally lead to the propagation of creep macro-cracks.

In the present study the continuum damage mechanics is applied to assess the creep damage behavior. Special attention has been addressed to the influence of the creep damage accumulation on the on creep-fatigue crack growth rate. The interpretation of the experimental creep crack growth rate data at elevated temperature on $\mathrm{C}(\mathrm{T})$ specimens was given in terms of introduced creep stress intensity factors based on undamaged and damaged stress/strain rate fields. Comparison between the creep-fatigue crack growth rate characteristics indicates that they are a function of the continuum damage mechanics parameters.

\section{Stress based model}

Let $\omega$ denote in Eq.(1) the measure of damage with $\omega=0$ denoting the undamaged state and $\omega=1$ the fully damaged state. The creep strain rate accumulation constitutive equation is generalized by the authors $[1,2]$ to multi-axial state of stress using $J_{2}$ - flow theory as follows

$$
\frac{d \varepsilon_{\text {eqv }}}{d t}=\frac{3}{2} B \sigma_{\text {eqv }}^{n-1} S_{i j}\left(\frac{1}{1-\omega}\right)^{n}
$$

where $B$ and $n$ are constants of the Norton power law equation. The simplest creep damage rate model, which is introduced by Kachanov [1], is a function of applied nominal stress and current accumulated damage has the following form

$$
\frac{d \omega}{d t}=C\left[\sigma_{f}^{( \pm)} /(1-\omega)\right]^{m}
$$

where $C$ and $m$ are material constants. Similarly Eq.(2), Shlyannikov and Tumanov [3] introduced a model for the rate of accumulation of stress-based creep damage as a function of multi-axial stress function $\sigma_{f}^{( \pm)}$described by Eqs. $(3,4)$ in the generalized form

$$
\begin{gathered}
\sigma_{f}^{( \pm)}=(1-\chi) \sigma_{k k / m p}^{( \pm)}+\chi \sigma_{e}, \sigma_{e}=\left[\frac{3}{2} S_{i j} S_{i j}\right]^{1 / 2} \\
\sigma_{k k}=\sigma_{1}+\sigma_{2}+\sigma_{3}=3 \sigma_{m}, \quad S_{i j}=\sigma_{i j}-\sigma_{m} \delta_{i j}
\end{gathered}
$$

where $\chi$ is the experimental material constant that is determined as the ratio of uniaxial tensile to compression strength $\chi=\sigma_{t} / \sigma_{c}$. For brittle fracture $\chi=0$, while for ductile fracture $\chi=1$. Going back to Eq.(3) it should be noted that in Eq.(2) $d \omega / d t$ is assumed to be implicit function of the hydrostatic stress $\sigma_{m}$ and the von Mises equivalent stress $\sigma_{e}$. The governing parameter in the form of the material constant $\chi$ helps in describing the

Corresponding author: shlyannikov@,mail.ru 
effect of the multi-axial state of the stress-strain behavior of the material.

\section{Governing parameters}

To interpret the experimental data for the creep-fatigue crack growth rate in the $\mathrm{C}(\mathrm{T})$ specimen, the creep stress intensity factor (SIF) values are used based on the experimentally measured load-line deflection rate [4,5]

$$
\bar{K}_{\sigma r}=\left[\frac{P}{\sigma_{0} B I_{n}^{F E M}(\theta, t, n, \omega)} \frac{\dot{V}_{c}}{b W L}\left(\frac{f^{\prime}}{f}\right)\right]^{\frac{l}{n+1}}
$$

where $\dot{V}_{c}$ - is the force-line displacement rate, $b$ - is specimen thickness and $f$ - is geometry dependent correction factor, $W$ is specimen width, $L$ is characteristic size. Equation (5) for the creep stress intensity factor includes a governing parameter in the form of $I_{n}$-integral, generally, for three-dimensional creeping solids. Shlyannikov et al. [4] modified the singular asymptotic fields in terms of the creep SIF under the secondary creep conditions to obtain the stress, strain, and displacement rates for elastic-nonlinearviscous materials. Moreover, a numerical method was introduced [4] to determine the governing parameters of the creep-crack tip fields in terms of the $I_{n}$-integral for power-law creeping materials. This method was extended to analyze the creep-damaged material-fracture resistance characteristics. According to this approach, the $I_{n}$-integral value can be obtained directly from the FEA distributions of the dimensionless angular stress $\tilde{\sigma}_{\#}^{1 E M}$ and displacement rate $\dot{\tilde{u}}_{i}$ functions

$$
I_{n}^{F E M}(\theta, t, n, \omega)=\int_{-\pi}^{\pi} \Omega^{F E M}(\theta, t, n, \omega) d \theta
$$

$$
\begin{aligned}
& \Omega^{\text {FTM }}(\theta, t, n, \omega)=\frac{n}{n+1}\left(\tilde{\sigma}_{p}^{F T M}\right)^{n+1} \cos \theta-
\end{aligned}
$$

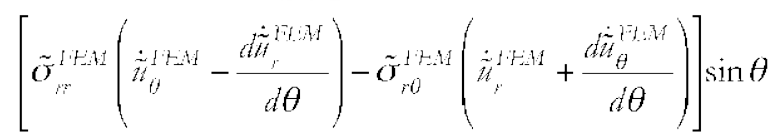

$$
\begin{aligned}
& -\frac{1}{n+1}\left(\tilde{\sigma}_{H}^{F \Gamma M} \dot{\tilde{u}}_{r}^{F W H}+\tilde{\sigma}_{r \theta}^{F W} \dot{\tilde{u}}_{\theta}^{F F M}\right) \cos \theta
\end{aligned}
$$

where $t$ is creep time, $\tilde{\sigma}$ is the von Mises equivalent stress and $\bar{\sigma}_{e}^{F E M}=\sigma_{e}^{F E M} / \sigma_{0}$. The numerical parameter $I_{n}$-integral and $\theta$-variation angular functions of the suitably normalized functions $\tilde{\sigma}_{i j}$ and $\dot{\tilde{u}}_{i}$ depend on the creep exponent $n$ and damage function $\omega$. Unlike the conventional models used to predict the creep-crack growth wherein the $I_{n}$-integral is a function of only the creep exponent, in the present study, it is demonstrated that the governing parameter depends on the creepdamage function and creeping crack-tip stress-strain rate fields.

The particular case of the $\mathrm{C}(\mathrm{T})$ specimen FE-steady state analysis carried out using constitutive equation (1) with $\omega=0$ ignores in the definition creep damage accumulation and growth. Since the stress and displacement rate ahead of the crack tip are a function only the creep time. Therefore post-processing governing parameters in the form of creep $I_{n}$-integral and creep stress intensity factor $K_{c r}$ were calculated by substituting numerical values of stress tensor components $\sigma_{i j}^{F E M}(\omega=0)$ and displacement rates $\dot{u}_{i}^{F M M}(\omega=0)$ into Eqs. $(5,6)$, respectively. This type of numerical results will denote as undamage fields. Accordingly, other types of basic calculations are performed for the elastic-nonlinear-viscous material with damages (Eq.1) that depend on the multi-axial of stress of state. In these cases the numerical values of stress tensor components $\sigma_{i j}^{F E M}(\omega, \chi)$ and displacement rates $\dot{u}_{i}^{F E M}(\omega, \chi)$ again were substituted into the left part of into Eqs. $(5,6)$, respectively. As a result, the values of the post-processing governing parameters in the form of creep $I_{n}$-integral and creep stress intensity factor $K_{c r}$ were determined. This type of numerical results will denoted as damage fields.

\section{Results and discussion}

Full-field FEA are performed using ANSYS finite element (FE) code to study the damage effect on the crack-front stress fields for $\mathrm{C}(\mathrm{T})$ specimen (Fig.1). Along the thickness direction, an identical planar mesh is repeated from the symmetry plane to the free surface. To catch the drastic change of the stress field near the free surface, the thickness of successive FE layers is exponentially reduced from the mid-plane toward the free surface. In the circumferential direction, 40 equally sized finite elements are defined in the angular region from 0 to $\pi$. The size of each ring gradually increases with the radial distance from the crack tip. The radial sizes of the finite elements are varied according to the geometric progression. Typical FE-mesh of the $\mathrm{C}(\mathrm{T})$ specimen contains 71640 elements. According to the symmetrical properties, one quarter of the actual structure was selected to establish the three-dimensional finite element model. The twenty-node quadrilateral brick isoparametric three-dimensional solid elements were used to model for the $3 \mathrm{D} C(\mathrm{~T})$ specimen configuration.

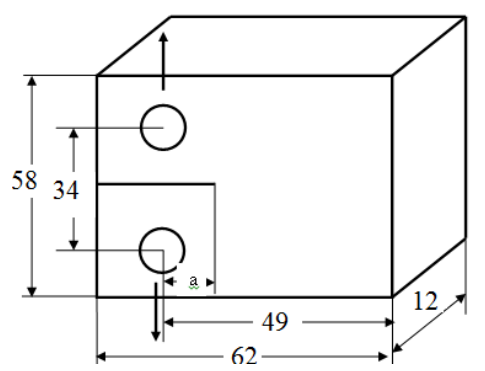

Fig. 1. Geometry of $\mathrm{C}(\mathrm{T})$ specimen. 
By employing the constitutive Eq. (1) directly into the FEM rate-dependent formulation and using an explicit time integration procedure, we obtain a standard Runge-Kutta integration scheme wherein the finiteelement stiffness matrix is derived from the elastic moduli. Thus, to determine the numerical stress/strainrate fields, we need to first determine the damage-rate function by substituting the stress obtained in the initial iteration into Eq. (2). After determining the damage function $\omega$ using numerical integration and by substituting the creep strain rate $\dot{\varepsilon}_{i j}$ of Eq. (1) obtained from the resulting damage function $\omega$ into the ANSYS, the damage stress/strain rate fields are found. Finally, after obtaining the solution to the nonlinear problem, the ANSYS output file is used as an input data for the special code developed to determine the dimensionless stress-strain angular distributions, damage contour, and creep-stress intensity factor.

The numerical calculations and tests material is $12 \mathrm{Cr} 1 \mathrm{MoV}$ steel which is used for main power plant components such as steam piping and reheat tube. The creep properties of analyzed material at the elevated temperature of $550^{\circ} \mathrm{C}$ are summarized in Table 1 .

Table 1. The creep properties of material.

\begin{tabular}{|c|c|c|c|c|c|}
\hline $\begin{array}{c}\mathrm{E} \\
{[\mathrm{GPa}]}\end{array}$ & $\begin{array}{c}\sigma_{0} \\
{[\mathrm{MPa}]}\end{array}$ & $\mathrm{B}$ & $\mathrm{n}$ & $\mathrm{C}$ & $\mathrm{m}$ \\
\hline 200 & 100 & $1 \times 10^{-14}$ & 5.0 & $1 \times 10^{-10}$ & 3.0 \\
\hline
\end{tabular}

\subsection{Creep-crack front stress fields}

The important factor in this part of the present study is the influence of the realistic three-dimensional stress-strain state of the finite size cracked body on the creep-damage process. Hence, 3D full-field FE-analysis was performed on the standard compact type, C(T) specimen containing a crack subjected to pure mode I fracture. The specimen dimensions chosen are as recommended by the ASTM test standards with width $w$ $=49 \mathrm{~mm}$ and thickness $b=12.5 \mathrm{~mm}$. To estimate the stress fields and damage variations, the couple deformation and damage constitutive equations of the form $\sigma(\dot{\varepsilon}, \omega, \chi)$ (Eq. (1)), which include the proposed stress-based failure criterion (Eqs. (3) and (4)) are employed. The governing parameter $\chi$, which is the ratio of the uniaxial tension strength to the compression strength, is equal to 0.8 . These creep-damage constitutive equations are then incorporated into a finiteelement calculation to study the influence of the damage on the crack-tip fields. For the creep in undamaged and damaged materials, we consider the stages of creep time varying from $t=1 \mathrm{~h}$ up to $t=10^{3} \mathrm{~h}$ at the elevated temperature of $550^{\circ} \mathrm{C}$. In the following figures, the distribution of stresses and damages will be presented in the thickness direction along the crack front of the $\mathrm{C}(\mathrm{T})$ specimen (Fig.1) from the free surface $z / b=0.0$ to the mid-plane $z / b=0.5$.
To assess the effects of damage formulation, comparisons under extensive-creep conditions $t / t_{T}=45.7$ are made in Fig. 2 between the undamaged creep field and defective fields at the crack tip in the $\mathrm{C}(\mathrm{T})$ specimen. Fig. 2 shows the numerical dimensionless equivalent von Mises stress $\tilde{\sigma}_{e}$ angular distributions in the equivalent stress $\tilde{\sigma}_{e}$ at a crack tip distance of $\bar{r}=1.384 \cdot 10^{-3}$ (which indicate as R1) as a function of the crack front position in the thickness direction. Near the crack tip, the influence of the damage degree is evident and a sufficient difference is observed between the undamage and the creep damage fields. Furthermore, it is no longer true that $\theta$-variations in the dimensionless stresses are independent of the creep-damage parameter $\omega$. Moreover, the data presented in Fig. 2 demonstrate the 3D-effect of the finite thickness on the creep-damage field behavior at the crack tip. It follows from the comparison that in the undamaged material, the maximum of equivalent stress $\tilde{\sigma}_{e}$ is always located ahead of the crack $\theta=0$, whereas in the damaged material the maximum stress depends on the position along the crack front $z / b$.
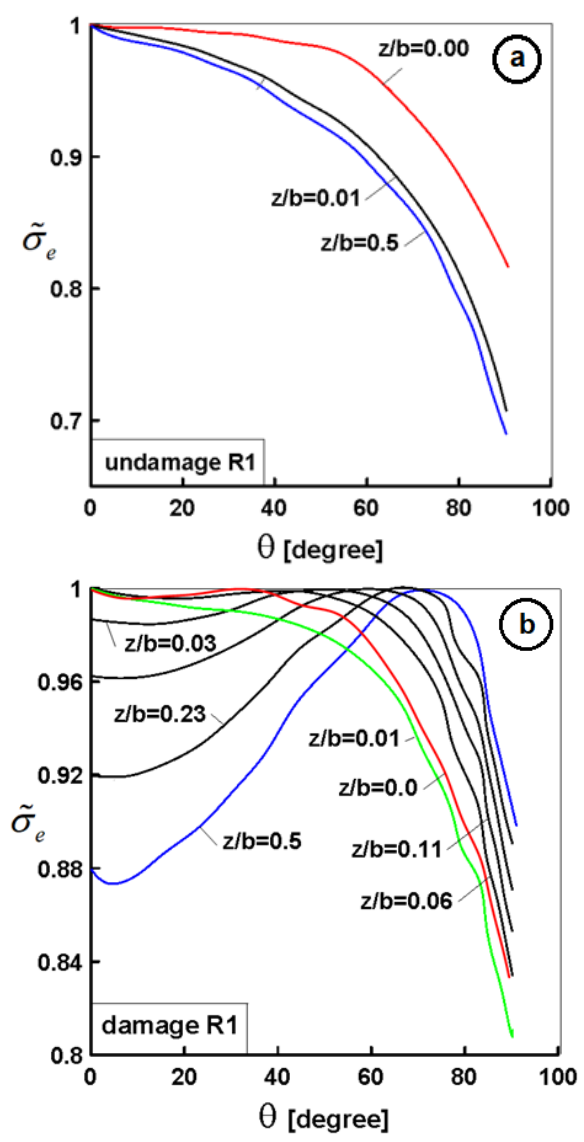

Fig. 2. Comparison dimensionless equivalent stress distributions for (a) undamaged and (b) damaged state.

\subsection{Creep-damage zone contour and size}

Figs. 3 and 4 show the contours of the creep damage zones around the crack tip and the maximum 
size of the creep damage zone behavior along of the crack front in the $\mathrm{C}(\mathrm{T})$ specimen, respectively, at creep time $t / t_{T}=45.7$ for the different value of crack tip distance. Fig. 3 shows that the 3D stress-strain state affects the shape and size of the creep-damage contours.

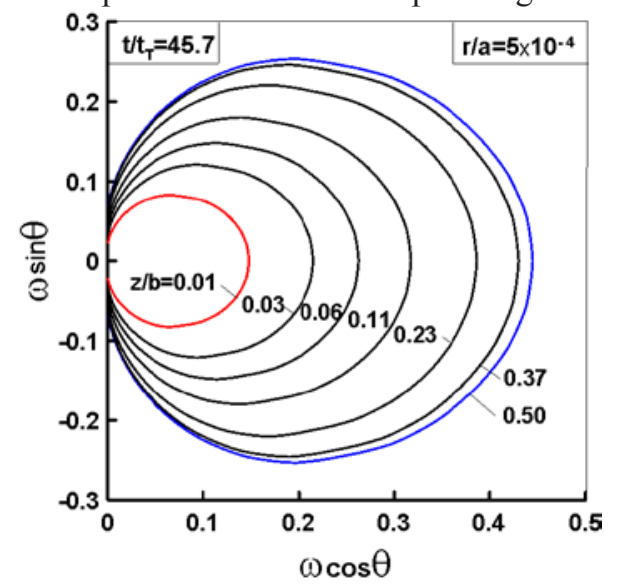

Fig. 3. Creep damage contour distributions along crack front.

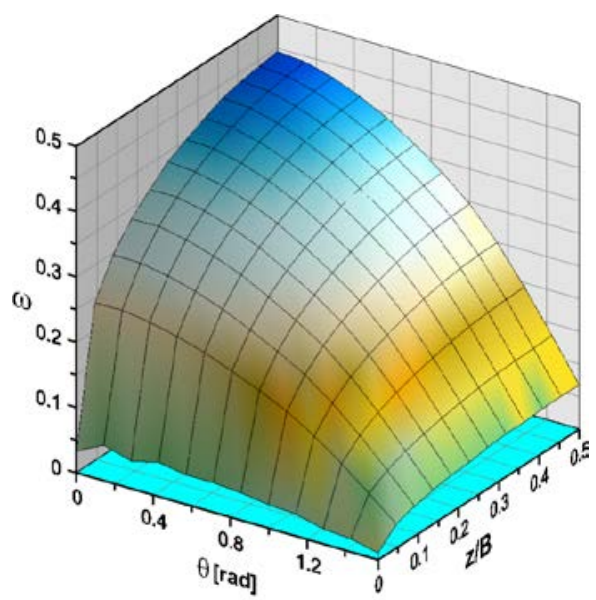

Fig. 4. Creep damage zone size distributions along crack front.

Moreover, the calculated contours strongly depend on the $\mathrm{C}(\mathrm{T})$ specimen thickness described by the dimensionless distance $z / b$. The shapes of the damages zones at the crack front distance $r / a=5 \times 10^{-4}$ in Fig. 4 show that the maximum damage contour boundary from the crack front is located along the crack line. However, under the distance $r / a=1.5 \times 10^{-3}$ the maximum size of the creep-damage zone is located approximately $\theta=60^{\circ}$ from the crack line ahead of the crack tip $\left(0^{\circ}\right.$ direction $)$.

\section{3 $I_{n}$-integral and creep SIF distributions}

As it follows from numerical results, the main effects of the damage parameter $\omega$ and material properties $\chi$ on the dimensionless stress components $\tilde{\sigma}_{j}$ and displacement rate components $\dot{\tilde{u}}_{i}$ arise through the normalizing factor in the form of the creep $I_{n}$-integral (Eqs. (6).
Figure 5 shows comparison creep $I_{n}$-integral distributions along the crack front for the stationary crack with dimensionless size $a / w=0.5$. It should be noted that the values for creep $I_{n}$-integral do not coincide with those of the plane strain undamaged and damaged fields at the same creep time. Furthermore, in the frame of the creeping solids formulation, the $I_{n}$-integral distributions as a function of the creep time for undamaged (Fig.5,a) and defective materials (Fig.5,b) are different and these distinctions increase with the increase in the holding time. In the definition, the plane strain solution is not sensitive to the finite thickness effect. In contrast, the proposed model (Eqs. (6)) can be used to better describe the full $3 \mathrm{D}$ conditions. It is clear that in the creeping solids, the behavior of the governing parameter of the crack-tip field $I_{n}$-integral is very sensitive to the variation in the material constitutive equation formulation.

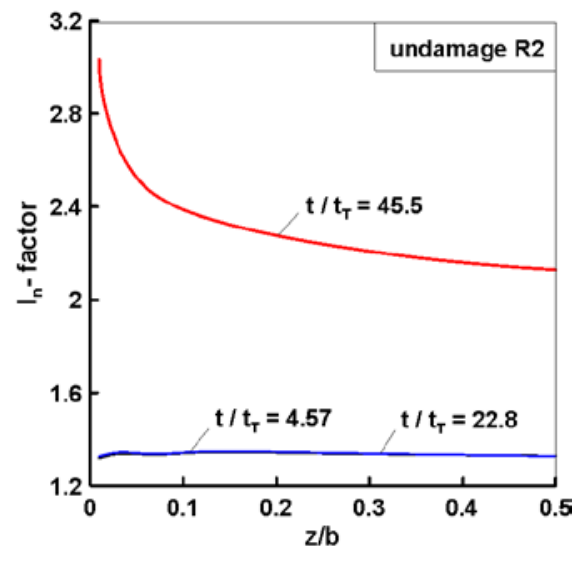

a)

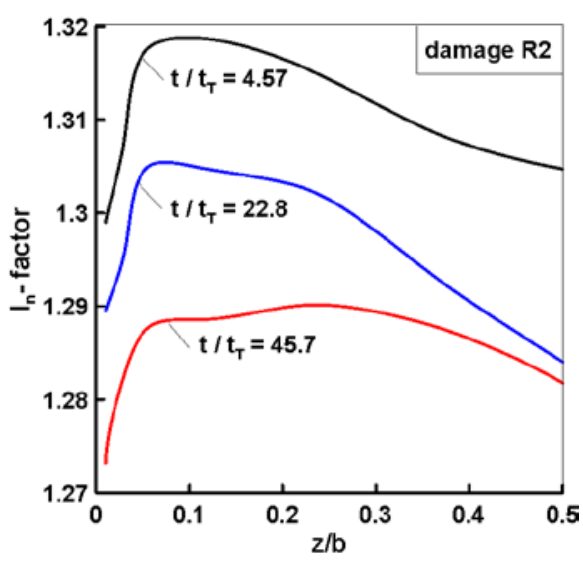

b)

Fig. 5. $\mathrm{I}_{\mathrm{n}}$-factor distributions for (a) undamaged and (b) damage state of stationary crack. 


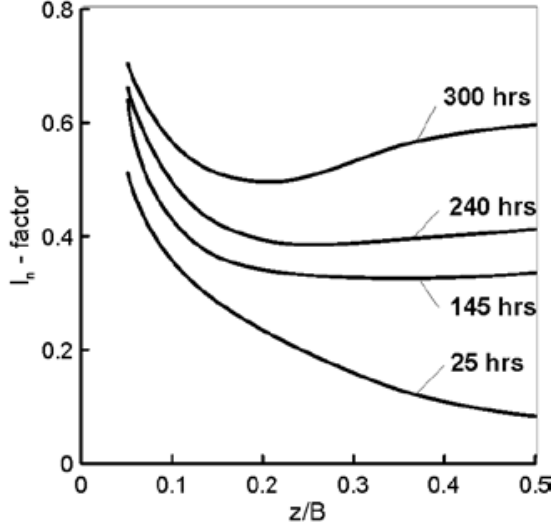

Fig. 6. $\mathrm{I}_{\mathrm{n}}$-factor distributions along growing crack front.

The behavior of the governing parameter of the power-law creep crack-tip fields $I_{n}$ for the $\mathrm{C}(\mathrm{T})$ specimen is plotted in Fig.6 along the growing crack front towards the thickness direction for damage state. The results are presented in a series of plots at three creep times. The first is $t=25 \mathrm{hrs}$ close to the transition time $t / t_{T}=1$, the second is $t=145 \mathrm{hrs}$, the third $t=240$ hrs and the fours $t=300 \mathrm{hrs}$, which are related to the extensive creep conditions. Fig. 6 represent the variation in the $I_{n}$-integral distributions through the creep crack growth stage for the crack front distance $\bar{r}=1.5 \cdot 10^{-3}$ (which indicate as R2). Based on Eq.(6), these distributions of the $I_{n}$-integral were used to calculate the creep-stress intensity factor in the $\mathrm{C}(\mathrm{T})$ specimen.

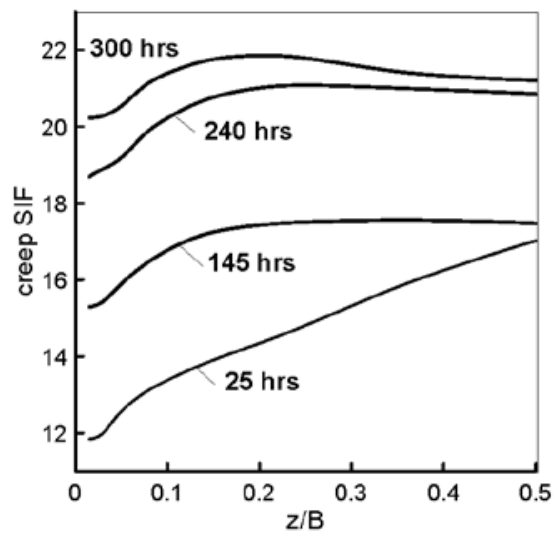

Fig. 7. Creep SIF distributions along crack front.

The distributions of the creep SIF along the crack front in the thickness direction is plotted in Fig.7 for the $\mathrm{C}(\mathrm{T})$ specimen. This parameter is plotted against the normalized specimen thickness $z / b$. The data presented in Fig.6 indicate that the level of the holding time has a significant impact on the distribution of the $\mathrm{I}_{\mathrm{n}}$-factor, and hence on the creep stress intensity factor. Recall that the position of each crack front corresponds to a certain number of loading cycles and the time creep. Analyzing the behavior of the creep SIF on Fig. 7 shows the influence of the crack front form through the $I_{n}$-factor as well as the value for the geometry dependent correction factor $\left(f^{\prime} / f\right)$ used in Eq. (5). It is possible to note that these distributions reproduce the experimentally observed crack front positions, i.e., the maximum value of the creep SIF is realized in the deepest point of the crack front whereas, the minimum of the creep SIF occurs in the surface point of the crack tip.

Fig. 8 shows the corresponding numerical results for the creep stress intensity factor behavior along the crack front in $C(T)$ specimen as a function of the creep time based on Eq. (5). In these figures, the finite element creep SIF variations correspond to that normalized using the crack tip distance used is $\bar{r}=1.384 \cdot 10^{-3}$. Fig. 8 shows the comparisons of the creep SIF distributions for undamaged elastic-nonlinear-viscous material with damage parameter in constitutive Eq. (1) $\omega=0$ and for damaged elastic-nonlinear-viscous material with damage parameter $\omega$ and constitutive law obtained using Eq. (2).

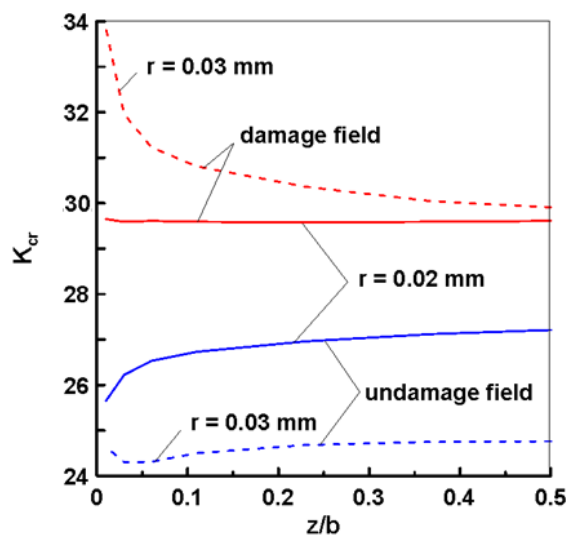

Fig. 8. Creep SIF behavior for undamaged and damaged state.

As observed in Fig.8, the creep SIF distributions as a function of the creep time for undamaged and defective materials are different and these distinctions depend on the crack front distance. Based on this comparison, we can conclude that the stress-based creep damage function (Eq. 2) provides a more accurate description of the actual near-tip fields for the elastic-nonlinear-viscous material.

\subsection{Creep-fatigue crack growth rate}

The description of the crack growth behavior under creep-fatigue conditions can be described in the terms of the elastic stress intensity factor $K_{I}$ or the magnitude of the creep stress intensity factor $K_{c r}$. The elastic SIF is strictly valid only for linear elastic behavior, but it can be used as an approximation if the creep zone size near the crack tip is limited. Further, the linear fracture mechanics approach for characterizing creep-fatigue crack growth behavior relied on $K_{l}$ for characterizing the crack growth rate per cycle, $d a / d N$, while maintaining the loading frequency and the loading waveform constant. To use the elastic and creep stress intensity factors to characterize the creep crack growth of the tested specimens, it is necessary to measure crack length increment as a function of number of loading cycles.

The tests were conducted in trapezoidal waveform for constant triangular loading/unloading portions with hold times superimposed at maximum load. The potential drop (PD) and the unloading compliance 
methods were used to monitor crack length during the creep-fatigue tests. The waveforms for the loading and unloading portions were trapezoidal, and the loading/unloading times were held constant ( $5 \mathrm{~s}$ rise and decay times). A hold time of predetermined duration, 60 $\mathrm{s}$, was superimposed on the trapezoidal waveforms at maximum load.

The tests were carried out at $550^{\circ} \mathrm{C}$ on special hightemperature test equipment with a load ratio, $R$, of 0.1 and maximum load values, $P_{\max }$, of 7 and $8 \mathrm{kN}$. The $\mathrm{C}(\mathrm{T})$ specimens were pre-cracked to an initial crack length to width ratio $a / W$ of approximately 0.4 under cyclic loading at room temperature. Two times during the total creep-fatigue life was the test was stopped, and the $\mathrm{C}(\mathrm{T})$ specimen cooled to room temperature; then the specimen was again subjected to creep-fatigue loading at $550^{\circ} \mathrm{C}$. This practice helped to determine the intermediate crack front positions during the creepfatigue loading between the initial (pre-crack) and the final crack fronts. After total failure of the specimen, measurements of the crack sizes were taken for four positions of the crack front by means of an optical microscope. For each front, the crack size at five equally spaced points centered on the specimen mid-thickness line was measured along the crack front. The measured values of crack length on the free surface $a_{f s}$ and at the mid-plane section $a_{m p}$ of the specimen are related to the corresponding number of loading cycles and creep times.

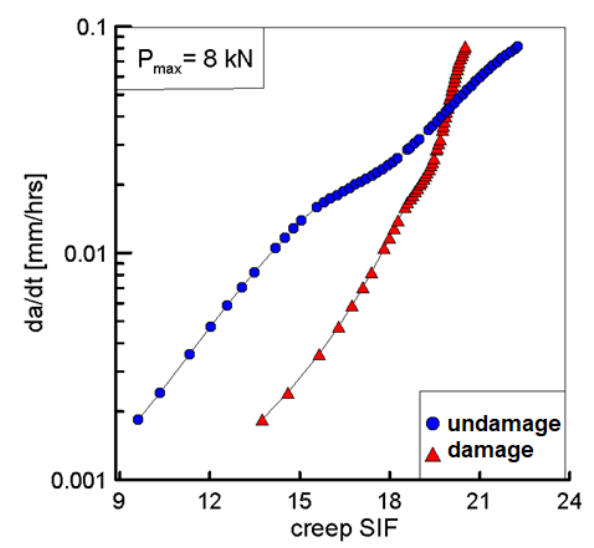

Fig. 9. Crack growth rate for different damage state.

Figure 9 represents the crack growth rate behavior of the $12 \mathrm{Cr} 1 \mathrm{MoV}$ steel at $550^{\circ} \mathrm{C}$ as a function of the dimensionless creep stress intensity factor in the form of Eq.(5) for the hold time 60s. It is seen that the experimental data fall within a relatively narrow scatter band. Again the dependence of the crack growth rate, $d a / d t$, on $\overline{K_{c r}}$ follows a nonlinear trend on a log-log scale at the final stage of creep-fatigue failure. To calculate the values of the creep SIF, the values of $I_{n^{-}}$ factor were used according to Eq. (6) and the geometry dependent correction factor $\left(f^{\prime} / f\right)$ corresponding to the intersection point of the crack front with a free surface of the sample.

In general, as expected, the crack growth rate increases with an increase in the elastic stress intensity factor, $\mathrm{C}(\mathrm{t})$-integral and creep stress intensity factor range. An overall reasonable correlation between the crack growth rate and $\Delta \mathrm{K}$ is an indication that dominantly quasi-elastic conditions were maintained throughout the testing. However, there is crack growth retardation at the final stage of failure due to blunting of the crack tip and high plasticity of the $12 \mathrm{Cr} 1 \mathrm{MoV}$ steel at elevated temperature. These are limitations regarding the use of $\Delta \mathrm{K}$ at an elevated temperature. It should be noted that the interpretation of the crack growth rate in terms of creep SIF permits better describe the impact of the material structure and properties on the crack growth resistance characteristics.

On Figure 9 one of lines is related to the undamage stress/strain rate state of the compact tension specimen, and the other belongs to the damage stress/strain rate fields along the crack front in the thickness direction. Unlike the definition for the elastic SIF and $C(t)$ parameter, the creep stress intensity factor approach gives the possibility to obtain the crack growth rate as a function of the creep constitutive equation formulation for the $\mathrm{C}(\mathrm{T})$ specimen. The comparison shows that the crack growth rate along the crack front is different.

Comparison between the creep-fatigue crack growth rate characteristics indicates that they are a function of the continuum damage mechanics parameters. The application of the introduced parameters through experimental study of the creep-fatigue crack growth rate confirms effectiveness of the proposed approach based on stress damage model.

The authors gratefully acknowledge the financial support of the Russian Science Foundation under the Project 17-19-01614.

\section{References}

1. L.M.Kachanov, Introduction to Continuum Damage Mechanics (Martinus-Nijhoff, Dordrecht, 1986)

2. J.Lemaitre, A Course on Damage Mechanics, (Springer-Verlag, Berlin, 1996)

3. V.N.Shlyannikov, A.V.Tumanov, Fatigue Fract. Eng. Mater. Struct., DOI: 10.1111/ffe.12766 (2018)

4. V.N.Shlyannikov, A.V.Tumanov, N.V.Boychenko, Engng. Fract. Mech. 142, 201-219 (2015)

5. V.N.Shlyannikov, A.V.Tumanov, N.V.Boychenko, A.M.Tartygasheva, Int. J. Press.Vess.Piping 139-140, 86-95 (2016) 